\title{
Decision-making in the ventral premotor cortex harbinger of action
}

\author{
Jose L. Pardo-Vazquez ${ }^{1}$, Isabel Padron ${ }^{1,2}$, Jose Fernandez-Rey ${ }^{2}$ and Carlos Acuña ${ }^{1}$ * \\ ' Laboratorios de Neurociencia, Facultad de Medicina, Departamento de Fisiología, and Complejo Hospitalario Universitario, Universidad de Santiago de \\ Compostela, Santiago de Compostela, Spain \\ ${ }^{2}$ Grupo de Procesos Cognitivos y Conducta, Facultad de Psicología, Universidad de Santiago de Compostela, Santiago de Compostela, Spain
}

\section{Edited by:}

Agnes Gruart, University Pablo de Olavide, Spain

\section{Reviewed by:}

Ranulfo Romo, Universidad Nacional

Autónoma de México, Mexico

Emilia lannilli, University Hospital

Dresden, Germany

\section{*Correspondence:}

Carlos Acuña, Laboratorios de Neurociencia, Facultad de Medicina, Departamento de Fisiología,

Universidad de Santiago de

Compostela, c/San Francisco, 1,

Santiago de Compostela E-15705,

Spain.

e-mail: carlos.acuna.castroviejo@ usc.es
Although the premotor (PM) cortex was once viewed as the substrate of pure motor functions, soon it was realized that it was involved in higher brain functions. By this it is meant that the PM cortex functions would better be explained as motor set, preparation for limb movement, or sensory guidance of movement rather than solely by a fixed link to motor performance. These findings, together with a better knowledge of the PM cortex histology and hodology in human and non-human primates prompted quantitative studies of this area combining behavioral tasks with electrophysiological recordings. In addition, the exploration of the PM cortex neurons with qualitative methods also suggested its participation in higher functions. Behavioral choices frequently depend on temporal cues, which together with knowledge of previous outcomes and expectancies are combined to decide and choose a behavioral action. In decision-making the knowledge about the consequences of decisions, either correct or incorrect, is fundamental because they can be used to adapt future behavior. The neuronal correlates of a decision process have been described in several cortical areas of primates. Among them, there is evidence that the monkey ventral premotor (PMv) cortex, an anatomical and physiological well-differentiated area of the PM cortex, supports both perceptual decisions and performance monitoring. Here we review the evidence that the steps in a decision-making process are encoded in the firing rate of the PMv neurons. This provides compelling evidence suggesting that the PMv is involved in the use of recent and long-term sensory memory to decide, execute, and evaluate the outcomes of the subjects' choices.

Keywords: premotor ventral cortex, decision-making, working memory, outcomes, event-related potentials

\section{GENERAL}

The discovery that the neuronal activity of the premotor (PM) cortex correlates with all the events that lead to a behavioral decision and with the outcomes of that decision has represented a major advance in our knowledge of the role of this area of the frontal cortex (Hernandez et al., 2002; Romo et al., 2004; Pardo-Vazquez et al., 2008, 2009; Lemus et al., 2009; Acuña and Pardo-Vazquez, 2011). These findings were preceded by clinical and experimental lesions in subhuman primates that ascribe the premotor cortex (PM or area 6 of Brodmann) a causative role in the so-called "syndrome of the premotor cortex," which included, forced grasping, spasticity, increase in tendon reflexes, and vasomotor disturbances in the upper extremity, contralateral to the lesion (review in Kennard et al., 1934). Jacobsen (1931) noted that a chimpanzee trained in feeding-box problems, after a fugitive paralysis resulting from ablation of PM cortex had passed, appeared unable to organize the necessary manipulations and had to relearn them. On commenting on the "syndrome of premotor cortex" Walshe went on to call its characteristic symptom "an intellectual deficit" because the subject has "forgotten how" to perform certain taught movements (Walshe, 1935).

Although these findings were difficult to interpret because the lesions involved adjacent cortical areas, they also gave some hints that suggested the PM was involved in higher-order aspects of motor control. More conclusive evidence came with the introduction of methodological and technical approaches combining operant conditioning with electrophysiological recordings in monkeys. These studies suggested the involvement of PM in planning and execution of complex movements, motor set, preparation for and sensory guidance of movements, reorganization of movements, and learning (Roland et al., 1980; Halsband and Passingham, 1982; Weinrich et al., 1984; Halsband and Freund, 1990; Mitz et al., 1991; Passingham, 1993; Kurata and Hoffman, 1994; Wise et al., 1997) as well as in complex relations with the immediate extrapersonal space (revised in Rizzolatti and Luppino, 2001) and perceptual decision-making (Romo et al., 2004; Pardo-Vazquez et al., 2008, 2009; Lemus et al., 2009). Additionally, conjunction analysis of fMRi data has suggested the involvement of the PM cortices in processing trigeminal information (Iannilli et al., 2007). These results can suggest functional heterogeneity and, in fact, the PM is architectonically heterogeneous and several subdivisions have been proposed on histological or hodological grounds (e.g., Matelli et al., 1985; Luppino and Rizzolatti, 2000; Petrides et al., 2005; Graziano and Aflalo, 2007) although a precise correlation between anatomy and function is not totally clear at the present time. This paper is focused on the ventral premotor (PMv) 
cortex where these clinical and experimental studies have led to the proposition that its neural apparatus together with its system connections generates the appropriate signals to decide, act, and record whether the behavioral outcomes were correct or incorrect. Moreover, in order to understand the PMv function we will refer not only to the PMv itself but we will also compare the results obtained in this area with those from other PM subdivisions as well as from other cortical or subcortical areas.

\section{THE PREMOTOR CORTEX AND THE CONTROL OF MOVEMENT}

Luria (1980) described that lesions of the PM cortex produced contrasting disturbances; on the one hand they produced disturbance "in automatic intellectual operations" such as the correct understanding of written texts, and, on the other hand, in the integration of complex motor acts. The "syndrome of the premotor cortex" was defined as a clinical entity, which included impairment of skilled movements of the fingers and vasomotor disturbances (Kennard et al., 1934). Interestingly, all manifestations of the condition can be reproduced experimentally in subhuman primates by lesions more or less restricted to the PM area and these symptoms are clinically different from those of the motor-area syndrome; for instance, lesions of the primary motor cortex (M1) or of the cortico-spinal fibers originating in M1 cause paresis, predominantly of finger movements (Denny-Brown and Botterell, 1948; Fulton, 1949; Travis, 1955; Fries et al., 1993). These symptoms can be due to disruption of connections of the PM with both the motor cortex and the motoneuronal pools in the spinal cord (Preuss et al., 1996; Cerri et al., 2003; Shimazu et al., 2004).

From then on many studies have focused on the motor-related properties of the PM cortex. Most of the work has been performed in the PMd, some included the PMd and PMv and some were unspecified. The role of the premotor medial (PMm) cortex in motor preparation has also been studied; it was found that this cortical area is involved in initiating hand movements both when they were self-initiated or externally timed (Romo and Schultz, 1987, 1992). Single extracellular unit recordings in the PM cortex support the hypothesis that the PMv is more involved than the PMd in the selection of an action or motor execution under visual guidance, while the PMd would be more involved in the preparation of movements (Weinrich et al., 1984; Passingham, 1993; Kurata and Hoffman, 1994; Wise et al., 1997; Rizzolatti and Fadiga, 1998). It has also been suggested that in the monkeys that perform in visuospatial tasks the PM plays a role in the selection of motor programs based on environmental context, thereby playing a role in motor learning (Halsband and Freund, 1990; Mitz et al., 1991; Di-Pellegrino and Wise, 1993). In fact, the PM lesions impaired monkeys' ability to associate visual information with particular movements, which is also in line with the proposal that the PM plays a role in the visual guidance of movements (Halsband and Passingham, 1982; Mushiake et al., 1991; Kurata and Hoffman, 1994). Brain imaging studies confirmed the involvement of the PMv in both temporal sequencing and visual guidance of movements (Schubotz and Von Cramon, 2001; Schubotz et al., 2003).

Based on cytoarchitectural and histochemical data the monkey's agranular frontal cortex was divided in seven areas (F1-F7; Matelli et al., 1985; Rizzolatti et al., 1998) of which, areas F4 (caudally) and F5 (rostrally) correspond to the PMv. There are anatomical and functional differences between PMd and PMv but their correlation is still largely unclear because only in a few cases have both areas been studied with the same experimental paradigm. The hodology of the monkey's PM cortex also confirmed the histological sectors and suggested different functions (Kurata, 1991; Rizzolatti and Luppino, 2001). F5 receives somatosensory inputs from the second somatic sensory area (SII), the PF (7b), and the anterior intraparietal (AIP) area (Godschalk et al., 1984; Matelli et al., 1986; Luppino et al., 1999), and visual inputs from the superior temporal sulcus (STS) through the inferior parietal lobe (area PF; Markowitsch et al., 1987; Wise et al., 1997; Rizzolatti and Matelli, 2003). Accordingly, Graziano et al. (1997) found that neurons in area F5 respond to both visual and tactile stimulation, and to proprioceptive inputs. Area F4 receives visual and somatosensory information from the MST and MT of the STS, and from the PEc and PFG of the posterior parietal cortex, respectively, through the ventral intraparietal (VIP) area; accordingly, microstimulation and single unit recordings in F4 have been correlated with arm and face movements (Rizzolatti et al., 1998). Based on the connectivity of the PM cortex, Rizzolatti and Luppino (2001) differentiate the parieto-dependent and the prefronto-dependent areas. The main extrinsic input to the parieto-dependent areas ( $F 1$, $\mathrm{F} 2, \mathrm{~F} 4, \mathrm{~F} 5)$ is from the parietal cortex and the intrinsic connections are with F1. These areas send fibers directly to the spinal cord contributing to the cortico-spinal tract. Altogether, these studies suggest that F1, F2, and F3 use somatosensory information while F5 uses somatosensory and visual information for action or sensory-motor transformations. Other functional differences between PMd and PMv were reported but without precise correlation with the parcelation of the PM cortex. For example, neurons in PMv reflect processes in extrinsic coordinates more often than neurons in M1 do (Kakei et al., 2001), and effector independent activity is more frequent in PMv than in PMd (Hoshi and Tanji, 2002). On the other hand, neurons in PMv present premovement activity less frequently than neurons in PMd do (Boudreau et al., 2001; Hoshi and Tanji, 2002). Hepp-Reymond et al. (1994, 1999) found PMv neurons whose activity co-varied with an external force in a precision grip task, a result consistent with the hypothesis that the PMv might participate in processing of movement dynamics. Movement dynamics, which are widely represented across the motor areas of the frontal lobe, are less precisely represented in the PMd and PMv, thereby revealing areal specialization (Xiao et al., 2006). These findings are seen as a physiological counterpart to the anatomical findings of distinct PMv connectivity (He et al., 1993; Luppino et al., 1993; Boussaoud et al., 2005).

\section{REORGANIZATION OF MOVEMENTS AND MOTOR LEARNING}

These experiments highlighted differences between areas suggesting that the contribution of the PMv to motor planning and movement dynamics appears minimal but that they may play a role in planning visually guided movements, more specifically during conditional motor learning. This is exemplified by studies that have shown that the PM dorsal and ventral neurons respond differently to identical stimuli that instruct different actions (Boussaoud and Wise, 1993). Therefore, the PMv is involved in more than basic motor control. The studies that suggested that the 
$\mathrm{PM}$ areas can contribute to the functional recovery, reorganization, and motor learning of hand movements can be traced to the early ones of Jacobsen (1931). Based on the fact that lesions affecting non-primary motor areas cause predominantly higherorder motor disorders such as apraxia, other studies were focused on the role of the PM cortex in the reorganization of movements (Freund and Hummelshein, 1985; Halsband and Freund, 1990; Halsband et al., 1993; Passingham, 1993; Seitz et al., 1998; Carey et al., 2002; Fridman et al., 2004). In this line it was found that temporal interference with muscimol in $\mathrm{PMd} / \mathrm{PMv}$ provokes deficits in the use of non-spatial visual information to guide action (Kurata and Hoffman, 1994) and that after recovery of the MI cortex ibotenic acid lesions, injection of muscimol in the premotor (PMd/PMv) cortex of the affected side suppressed the recovery of the motor deficit (Liu and Rouiller, 1999). Also, Frost et al. (2003) demonstrated reorganization in the hand representation of primate ipsi-lesional PMv cortex associated with functional recovery. Particularly interesting are the studies that showed that PM lesions in monkeys made them unable to relearn a visual conditional motor task although they can see the cue or use the information from the cue (Halsband and Passingham, 1982, 1985; Petrides and Milner, 1982; Petrides, 1986). Patients with PM lesions were impaired in conditional motor learning when they have to recall a movement from memory on the basis of a sensory cue (Halsband and Freund, 1990). These results are consistent with the hypothesis that the PM cortex of the affected hemisphere can reorganize to control basic parameters of movement usually assigned to M1 function (Liu and Rouiller, 1999; Frost et al., 2003). Furthermore, learning-dependent changes in activity during a visual conditional task suggest for this area a role in the selection of movements on the basis of arbitrary associations (Mitz et al., 1991) in agreement with the proposal that PM plays a role in retrieval of movements from memory based on environmental context (Passingham, 1988). This view is supported by (a) reports of trans-cranial magnetic stimulation (TMS) of the PM cortex, which interfere with timing and motor learning and off-line consolidation (Schluter et al., 1998; Del Olmo et al., 2007; Boyd and Linsdell, 2009) and (b) by fMRi studies that show that recall from memory of an established motor skill shifts the activity from prefrontal regions to the PM, posterior parietal, and cerebellar cortices probably to stabilize the representation of the motor skill (Shadmehr and Holcomb, 1997; Amiez et al., 2006). Finally, a variety of studies made with fMRi, PET, TMS, and event-related potential (ERP) have shown increased activity in the PMv during training and learning and in selecting stimulus guided movements (Lang et al., 1992; Deiber et al., 1997; Shadmehr and Holcomb, 1997; Grafton et al., 1998; Schluter et al., 1998; Passingham and Toni, 2001; Kelly and Garavan, 2005) but not when performance was automatic, in agreement with the proposal for self-monitoring of ongoing movements (Hoshi and Tanji, 2000).

\section{COMPLEX SENSORY-MOTOR INTERACTIONS}

The experimental findings that the monkey's PMv could be involved in complex sensory-motor integrations come from the work of Rizzolatti and Craighero (2004). They described in area F5 neuronal activity related to complex movements and interactions between subjects, mirror neurons, and suggested that these processes are better revealed once the monkeys have understood the rationale of the behavior, which suggests a learning process. These qualitative studies have shown responses related to specific actions such as grasping or holding-tearing, which led to a proposal that the AIP-F5 circuit plays a role in visuomotor transformations for object grasping and manipulation while the VIP-F4 circuit will be involved in encoding the peripersonal space and in transforming the object location into appropriate movements (Jeannerod et al., 1995; Rizzolatti et al., 1998; Rizzolatti and Luppino, 2001; Rizzolatti and Craighero, 2004). The executive control attributed to the prefrontal cortex can be exerted on the PM cortex through the dense anatomical connections as well as with other cortical and subcortical structures such as the basal ganglia (e.g., Pandya and Barnes, 1987; Lu et al., 1994; Fuster, 1997). Therefore, the importance of the PM in higher-order aspects of the cerebral control of movement became evident.

As has been summarized, the role proposed for the PM in general and for the PMv in particular in different aspects of movement control has been obtained with different experimental approaches. Among them, the behavioral paradigms used have been conditional motor tasks, association tasks, or visual instructed delay tasks. In designing the tasks what was in mind was to reveal the participation of PM in movement planning, control, or execution, in the broad sense. These tasks have in common perception of sensory stimuli, motor decisions based on comparison of these stimuli and delay periods between them in which memory traces of these stimuli have to be maintained in working memory. Although all these findings obtained with the these tasks suggested a role for the PMv in cognitive processes that can range from perception to action the first experimental evidence came from the work of Romo et al. in the PMv and PMm cortices (Hernandez et al., 2002; Romo et al., 2004). They used an experimental approach that has proved very fruitful in studying the function of the posterior parietal and frontal cortices (Evarts, 1966; Fuster and Alexander, 1971; Mountcastle et al., 1975) and has allowed an identification of neurons in the PMv cortex that supports both perceptual decisions (Romo et al., 2004; Pardo-Vazquez et al., 2008, 2009; Lemus et al., 2009; Roca-Pardiñas et al., 2011) and correct and error monitoring (Pardo-Vazquez et al., 2008, 2009; Acuña et al., 2010).

\section{PERCEPTION, DECISION-MAKING, WORKING MEMORY, AND PERFORMANCE MONITORING}

The physiological and clinical data gathered during the last few years, including the pattern of connections of the PMv cortex, suggest that the PMv could participate in the processes that link sensory perception with motor reports, including the maintenance of information in working memory. In fact, as has been reviewed, the PMv receives inputs from sensory areas, association areas related to working memory and decision-making, and subcortical areas implied in behavioral monitoring, and sends projections to motor-related areas of the frontal cortex, subcortical structures, and spinal cord (Godschalk et al., 1984; Schell and Strick, 1984; Markowitsch et al., 1987; Keizer and Kuypers, 1989; Dum and Strick, 1991; He et al., 1993; Hoover and Strick, 1993; Lu et al., 1994; Ghosh and Gattera, 1995; Luppino et al., 1999; Rouiller et al., 1999; Mcfarland and Haber, 2002; Boussaoud et al., 
2005; Dancause et al., 2006; Borra et al., 2008). To study the participation of the PMv cortex in these processes, it was necessary to design behavioral tasks that include the putative cognitive steps that link perception with action. Perceptual decisions fulfill this prerequisite, as they require subjects to use the available sensory information to make a judgment and communicate it by a simple behavioral action. One kind of perceptual decision task, the two alternative-forced choice (2AFC) task, has the added advantage of allowing the study of neuronal correlates of maintenance of sensory information in working memory. In fact, since the pioneer works of Fuster and Kubota (Fuster and Alexander, 1971; Kubota and Niki, 1971) the tonic elevated neuronal firing rate during the delay period of a memory task is considered to be a neuronal correlate for the internal representation and storage of perceptual or motor information. Although the neuronal activity obtained in the PMv during the delay period of conditional motor tasks has not been explicitly described as working memory (e.g., Weinrich and Wise, 1982; Kurata and Wise, 1988) some could be memory-related activity. Learning-dependent changes in activity (Mitz et al., 1991) and spatial attention/memory and intention related activity have also been described in the PM cortex (Boussaoud, 2001).

\section{PREMOTOR VENTRAL CORTEX AND PERCEPTUAL DECISIONS}

The PM cortex was associated to perceptual decisions, for the first time, by Romo et al. (1993, 1997), who recorded the activity of single neurons from the PMm cortex while monkeys performed in a somatosensory categorization task. Later, the function of the PM cortex has been studied by recording the activity of single neurons from this area while monkeys (Macaca mulatta) performed perceptual discrimination tasks of different sensory modalities, including somatosensory (Romo et al., 2004), visual (PardoVazquez et al., 2008, 2009; Acuña et al., 2010; Roca-Pardiñas et al., 2011), and auditory (Lemus et al., 2009). In these tasks, subjects have to choose between different behavioral responses based on the comparison of two stimuli. Romo et al. (2004) followed this approach for the first time, using a discrimination task in which the monkeys had to compare the frequencies of two vibrotactile stimuli (S1 and S2) applied sequentially to the monkeys' fingertips and separated by a fixed delay period. They found that the $\mathrm{PMv}$ neurons encode all the cognitive steps necessary to solve the perceptual decision: the frequency of the first stimulus when it is presented and also when it is maintained in working memory; the frequency of the second stimulus; the comparison between the two stimuli and the motor commands expressing the result of the comparison. By recording the activity of these PMv neurons in a control task, the authors also ruled out a purely motor explanation of their findings (Romo et al., 2004).

The role of the PMv cortex in visual perceptual decisions has been studied with two variants of an orientation discrimination task (Figures 1A,B; Pardo-Vazquez et al., 2008, 2009; Acuña et al., 2010; Acuña and Pardo-Vazquez, 2011). In the continuous discrimination (CD) task, monkeys have to perceive the orientation of two lines ( $\mathrm{S} 1$ and $\mathrm{S} 2$ ) showed sequentially and separated by a fixed delay. Then, they have to compare the orientation of S2 with the orientation of the memory trace left by $\mathrm{S} 1$ and decide whether S2 was oriented to the left or to the right of S1. Finally, they have to communicate the result of their decision by making an

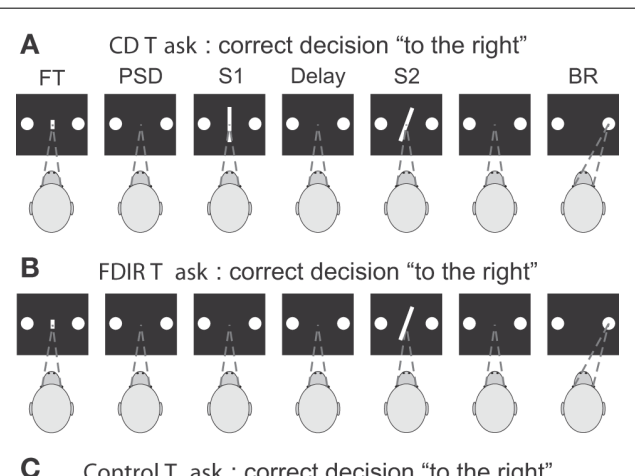

C Control T ask : correct decision "to the right"

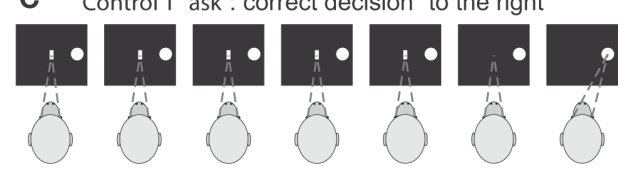

D

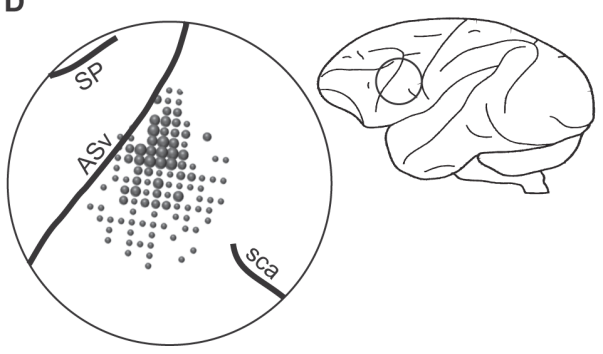

FIGURE 1 | Sequence of events during the two discrimination and the control tasks and localization of the recording area. (A) Continuous discrimination (CD) task: the fixation target (FT) and two circles appeared simultaneously in the center and at both sides of the monitor screen, respectively; the monkey initiated the trial by fixating the $\mathrm{FT}$; fixation had to be maintained during the trial, otherwise it was aborted; when the FT disappeared and after a variable prestimulus delay (PSD; 100-300 ms), two stimuli (S1 and S2), each of $500 \mathrm{~ms}$ duration, appeared in sequence separated by a delay of $1 \mathrm{~s}$; once the second stimulus had disappeared, the monkey made an eye movement (behavioral response, BR) to one of the two circles to indicate whether the orientation of the second stimulus was to the left or to the right of the first; correct discriminations were rewarded. (B) Fixed discrimination with implicit reference (FDIR) task: same temporal sequence and stimuli set as in the CD task, except that $\mathrm{S} 1$ was not shown (i.e., it was implicit) and had to be recovered from long-term memory by trial and error; the implicit stimulus changed from block to block of about 90 trials each and only S2 changed trial by trial. (C) Control task: the FT and one of the circles appeared simultaneously; the monkey had to maintain fixation until the FT had disappeared and made an eye movement to the circle; the duration of the trial was the same as that of the CD task. The interval between trials changed randomly in the three tasks between 1.5 and $3.5 \mathrm{~s}$. (D) Sketch of the brain showing the localization of the recording area. ASv, arcuate sulcus ventralis; SP, sulcus principalis; sca, sulcus subcentralis anterior. Adapted with permission from Pardo-Vazquez et al. (2008, 2009).

eye movement toward one of the response targets. If the response is correct, the monkeys are rewarded. In the fixed discrimination with implicit reference (FDIR) task, the first stimulus is not presented - it is implicit - and remains unchanged in a block of trials. At the beginning of each block, monkeys have to retrieve the correct implicit S1 from long-term memory (LTM) by trial and error. From then on the decision process continues as in the CD task (Vazquez et al., 2000; Pardo-Vazquez et al., 2009). 
To perform the comparison, the orientation of S1 has to be available during the presentation of S2 (i.e., the comparison or decision period). Therefore, S1 has to be maintained in working memory until S2 is presented. If the PMv neurons encode the sensory evidence used to reach a decision, it is expected that their firing rate will represent the orientation of S1 during the decision period. Besides, if these neurons have a role in the discrimination process, it is expected that they will represent the comparison of the two stimuli and/or the final choice (i.e., whether S2 was oriented to the left or to the right of S1). The dependency between the firing rate and (a) the orientation of $S 1$, (b) the difference between S2 and S1 (S2-S1), and (c) the choice [ $\operatorname{sign}(\mathrm{S} 2-\mathrm{S} 1)$ ] was assessed by means of stepwise linear regression (SLR) analysis on single neurons recorded in the PMv (Figure 1D). The use of a sliding window of $100 \mathrm{~ms}$ moving in $20 \mathrm{~ms}$ steps allowed for the study of the dynamic representation of the discrimination process throughout the trial. This analysis showed that the PMv neurons participate in encoding different components of the perceptual decision and that these representations evolve in time during both visual discrimination tasks (Figure 2; Pardo-Vazquez et al., 2008, 2009). Firstly, it has been found that neurons from the PMv encode the orientation of S1 in the CD task. These representations are not limited to the time interval during which the stimulus is presented but are also observable during the delay period and especially during the presentation of S2 (Figure 2A). Therefore, the PMv neurons encode the orientation of S1 during its presentation and also maintain it in working memory until the presentation of S2, when the comparison is made. Similar results were found in the FDIR task in which the orientation of S1 had to be recovered from LTM (Figure 2B): there are PMv neurons encoding the orientation of the implicit S1 from the beginning of the trial until the presentation of S2, when this information is used to compare the orientation of both stimuli. Secondly, it has been found that PMv neurons encode, during the first 200$300 \mathrm{~ms}$ of the comparison period, the difference between S2 and $\mathrm{S} 1$ in the CD and FDIR tasks. This result suggests that these neurons use the information about the orientation of S1 to compare it with the orientation of the second stimuli. Thirdly, there are PMv neurons that encode the choice, during the comparison period, in both the CD and FDIR tasks. This suggests that the activity of the PMv neurons is very close to the behavioral choices, as will be discussed later. Finally, it is worth noting that some PMv neurons encode the whole process that links sensory information with the behavioral action in the two variants of the discrimination task. The example neuron shown in Figure 3 encodes the orientation of $\mathrm{S} 1$ at the beginning of the decision period (i.e., when the second stimulus is presented and the comparison can be made); after a few tens of milliseconds the same neuron encodes the comparison of S2 and S1, and finally, this neuron also encodes the choice.

The results obtained in the somatosensory and visual discrimination tasks were confirmed in an auditory task (Lemus et al., 2009). Therefore, the PMv neurons encode the same information regardless of the sensory modality: somatosensory, auditory, or visual. There are neurons that represent the sensory features of the first stimulus, its memory trace, the comparison of the two stimuli and the choice. However, there is a difference between the

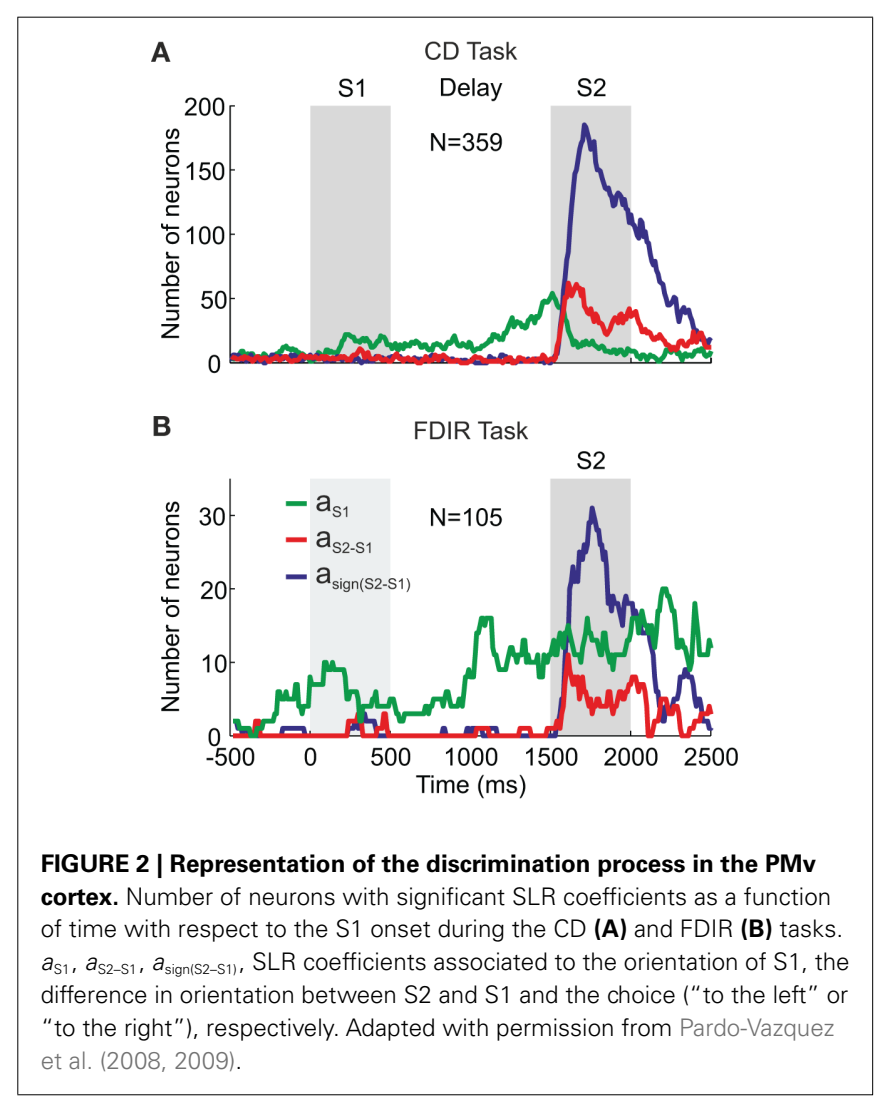

results obtained in the somatosensory and auditory modalities and those obtained in the visual discrimination tasks: the PMv neurons encode the sensory features of the second stimulus in the somatosensory and auditory tasks but not in the visual tasks (Romo et al., 2004; Pardo-Vazquez et al., 2008, 2009; Lemus et al., 2009). These discrepancies may be explained by the temporal differences between the stimuli used; the orientation of the lines in the visual tasks can be perceived as soon as the stimulus is presented while the frequency of the somatosensory and auditory stimuli has to be integrated over time.

\section{DISCRIMINATION CAPABILITY OF SINGLE NEURONS FROM THE PMv CORTEX}

Another relevant issue regarding the role of the PMv cortex in perceptual decisions is the number of neurons that are needed to perform the discriminations. On the one hand, if the discrimination capability of each PMv neuron were lower than the behavioral performance, this would mean that the information about the decision has to be carried out by combining the activity of a great number of neurons. On the other hand, if each neuron had a similar discrimination capability to that behaviorally observed, a small group of neurons would be able to perform the comparison. The capability of single neurons from the PMv cortex to discriminate the orientation has been studied using trial-to-trial methodology based on signal detection theory (SDT; Green and Swets, 1966; Macmillan and Creelman, 1991). Sensitivity $\left(d^{\prime}\right)$ was used as the index of discrimination capability and was estimated for both behavioral and neuronal data (Acuña and Pardo-Vazquez, 

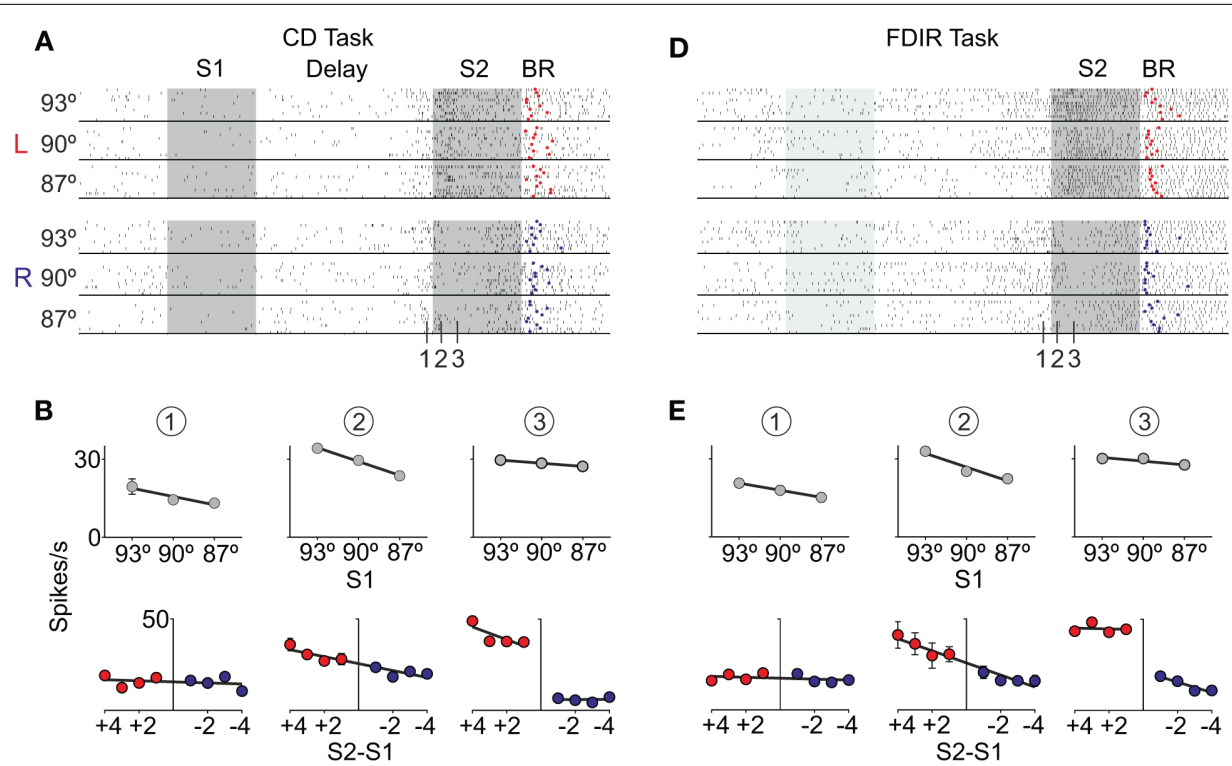

E
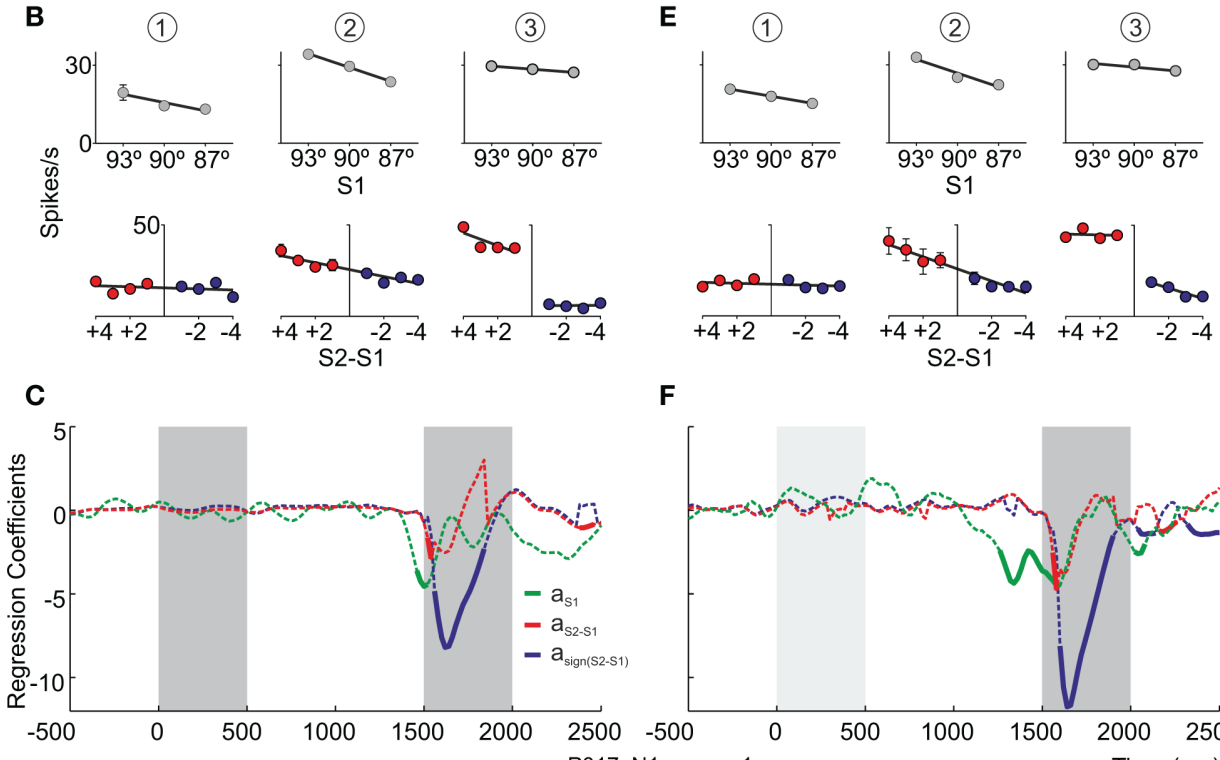

F

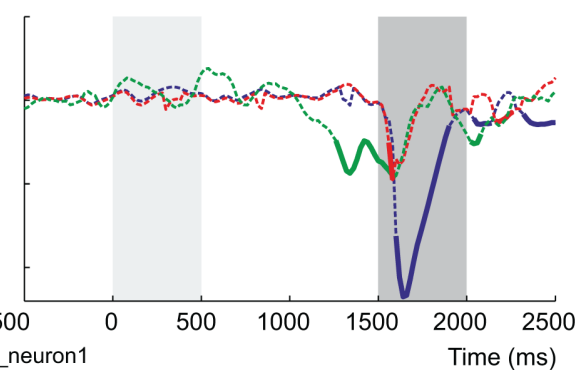

FIGURE 3 |The temporal evolution of neural activity depends on the trace left by the first stimuli - regardless of whether it was recently shown in the visual world or retrieved from LTM - on the comparison between the first and the second stimuli and on the choice. $(A, D)$ Response of the same neuron during the CD and FDIR tasks, respectively, sorted by $S 1\left(93^{\circ}, 90^{\circ}\right.$ and $\left.87^{\circ}\right)$ and the choice ( $L$, to the left; $R$, to the right). Raster plot: each line represents a trial and each tick an action potential. Trials were randomly presented. Red and blue dots signal the behavioral response $(B R)$ in each trial, to the left and to the right, respectively. (B,E) Temporal evolution of the average firing rates fitted as a function of: $\mathrm{S} 1$ and the relative orientation of S2 (S2-S1) during the delay (1) and the comparison (2 and 3) periods. (C,F) Stepwise Linear Regression coefficients, $a_{\mathrm{S} 1}, a_{\mathrm{S} 2-\mathrm{S} 1}$, and $a_{\text {sign(S2-S1), as a function of time; continuous traces indicate significant }}$ coefficients. Adapted with permission from Pardo-Vazquez et al. (2009).
2011). The main finding was that the sensitivity of a group of neurons from the PMv is close to the behavioral sensitivity (Figure 4). This suggests that subjects could obtain knowledge about their behavioral choices from the activity of a small number of neurons.

\section{CHOICE-RELATED SIGNALS IN THE PMv}

The fact that neuronal sensitivity is close to behavioral sensitivity does not imply the existence of a trial-to-trial covariation between the neural activity and the behavioral choices (Newsome et al., 1989). The analysis of the trial-to-trial covariation between the behavioral responses of the monkeys and the choices derived from the activity of single neurons (i.e., the neuronal choices) showed that, for some neurons, the coherence between behavioral and neuronal choices was close to 100\% (Figure 5; Acuña and Pardo-Vazquez, 2011). The relationship between neuronal activity in the PM cortex and behavioral choices trial-to-trial has also been studied in somatosensory and auditory discrimination tasks. The activity of the PMv neurons predicts the behavioral choices in about 75 and $90 \%$ of the trials in the somatosensory (Romo et al.,
2004) and auditory (Lemus et al., 2009) tasks, respectively. In the PMd cortex the percentage of predicted behavioral responses is about $60 \%$ and in the PMm it is about $70 \%$.

\section{Temporal evolution of the choice-related activity in the PMv cortex}

One of the main goals of studying the neural bases of decisionmaking is to understand how the neurons encode the decision in their firing rates and how these representations emerge and change in time as a function of different behavioral parameters of the discriminations. This question was addressed in the PMv cortex by assessing the optimal parameters (size and position) of the encoding window as a function of the difficulty (Acuña and Pardo-Vazquez, 2011; Figures 6A,B). This analysis showed that the percentage of coherence between neuronal and behavioral choices was higher than $85 \%$ for most PMv neurons (Figure 6C). The main results of the assessment of the optimal encoding window were that: (a) the PMv neurons encode the choices in a short time window (about $20 \mathrm{~ms}$ ) during the first 200-300 ms of the decision period (when S2 is presented) and (b) the optimal 


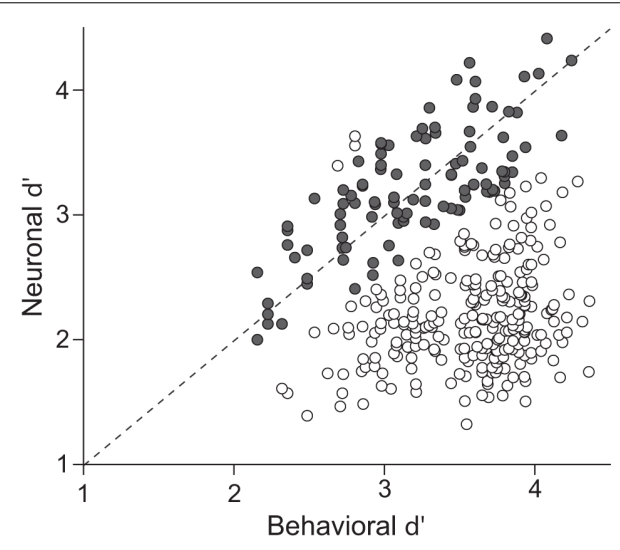

FIGURE 4 |The sensitivity of individual neurons from the PMv cortex is close to the behavioral sensitivity of the monkeys. Comparison of the neuronal and behavioral sensitivities for the 359 neurons with decision-related activity; solid circles correspond to neurons for which the ratio between behavioral and neuronal $d$ is between 0.8 and 1.2 ( $n=102$, $28 \%$ ); open circles correspond to neurons for which the ratio between behavioral and neuronal $d^{\prime}$ is lower than 0.8 or higher than 1.2 ( $n=256$, 72\%). Adapted with permission from Acuña and Pardo-Vazquez (2011).

parameters of the encoding window depend on the difficulty of the discrimination (Figure 7). The PMv neurons represent the easy discriminations earlier and within smaller encoding windows than the difficult ones. It is worth noting that to reveal the neuronal correlations of behavior each neuron should be analyzed individually both in the temporal evolution of its activity and in the size of the encoding windows. Given that the difficulty level of the decision affects the accuracy and timing of the behavioral choices, the parameters of the encoding window should also be adjusted to the difficulty level. Therefore, when using windows of fixed size or position for the analysis, the information encoded in the neuronal activity could be underestimated under certain conditions. In the somatosensory task it was found that the covariation between neuronal activity and behavioral choices during the stimulus presentation was higher for easier discriminations (Romo et al., 2004), while the level of coherence is almost equal for all difficulty levels in the CD task (Acuña and PardoVazquez, 2011). There is no reason to expect this dependency between the choice-related activity and the difficulty of the task, and this discrepancy could be related to the analytical procedure, as the covariation between neuronal activity and behavioral choices was analyzed with a fixed duration window in the somatosensory task.

\section{THE MOTOR COMPONENT OF THE TASK CANNOT EXPLAIN THE STRONG CHOICE-RELATED SIGNAL}

As the PMv neural activity has been associated with movement execution (Wise, 1985; Kakei et al., 2001), it could be possible that the neuronal activity, which was close to the behavioral choices, represented the motor component of the task. This possibility was ruled out by applying the same analyses to a subset of PMv neurons that were also recorded in a control task (Figure 1C). In this task, the motor component is the same as in the CD task but no discrimination (and therefore no perceptual decision) has to be

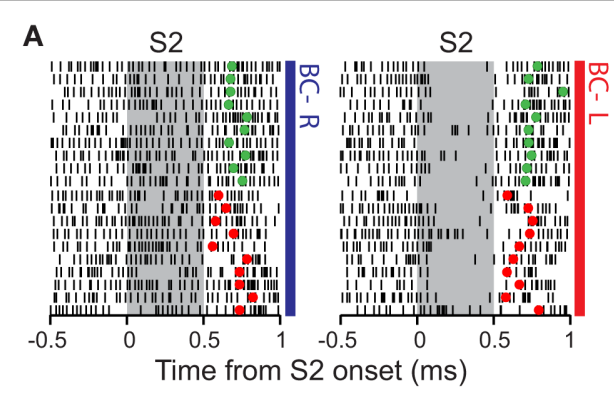

B

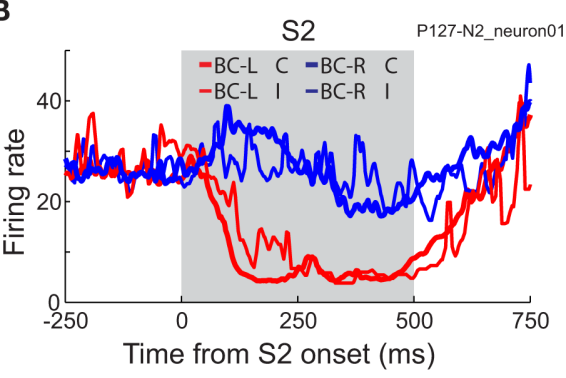

C

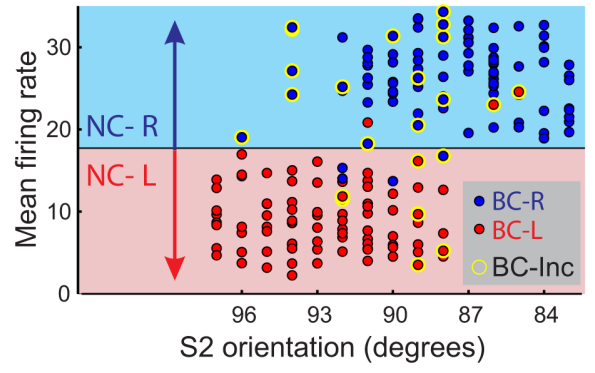

FIGURE 5 | The firing rate of individual neurons represents the behavioral choice of the monkeys both in correct and in incorrect trials. (A) Raster plot representing the activity of one neuron recorded during the CD task during the comparison period (S2). Trials are sorted by the behavioral choice of the monkeys to the left (BC-L) and to the right (BC-R) and by the correctness of the behavioral choice (Green and red dots, correct and incorrect behavioral choices, respectively). Trials were randomly presented. (B) Mean firing rate of the neuron in A, during the comparison period, for correct and incorrect behavioral choices to the left (BC-L C, BC-L I) and to the right (BC-R C, BC-R I). (C) For the same neuron, mean firing rate as a function of the orientation of $\mathrm{S} 2$ and the behavioral choice [red and blue dots represent trials in which the behavioral choices were to the left $(B C-L)$ and to the right (BC-R), respectively] during comparison period (S2). The firing rate in each trial is compared against different criteria to categorize neuronal responses as "to the left" or "to the right." The criterion (horizontal black line) is chosen to maximize the coherence between the neuronal and the behavioral choices. Red and blue background colors indicate the neuronal choices derived from the mean firing rate during the comparison period, to the left (NC-L) and to the right (NC-R), respectively. Yellow halos signal incorrect behavioral choices (BC-Inc). Adapted with permission from Acuña and Pardo-Vazquez (2011).

made. The results obtained in the control task were significantly different from those obtained in the CD task for both the sensitivity and the coherence (Figures 6A,B). This suggests that the choice-related activity of the PMv neurons does not represent the motor component of the discrimination task and that the choice itself is encoded in the activity of the PMv neurons (Acuña and 

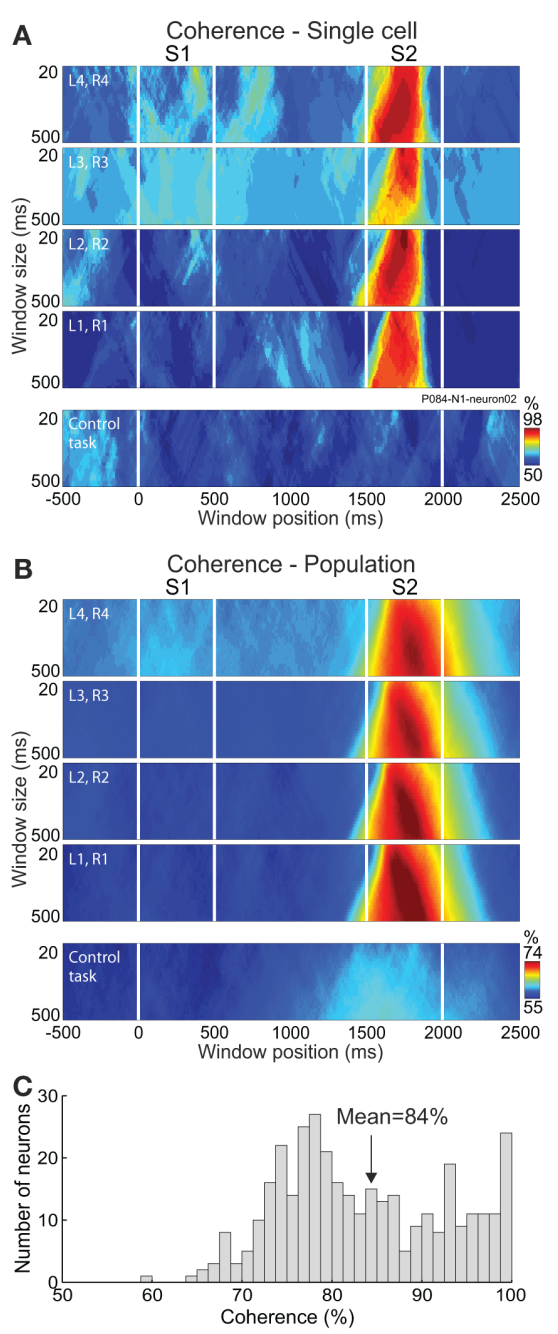

FIGURE 6 |The activity of PMv neurons predicts the behavioral choices of the monkey. $C D$ task: four upper tiers of each panel; Control task: lower tier of each panel. (A) For one example neuron each tier depicts the percentage of coherence between the neuronal and behavioral choices (color code) as a function of window position ( -500 to $2500 \mathrm{~ms}$ from S1 onset, in steps of $5 \mathrm{~ms}$; abscissa) and window size (20 to $500 \mathrm{~ms}$, in 20-ms steps; ordinate) for each level of difficulty [from the most difficult (L1, R1) to the easiest $(L 4, R 4)]$ in the $C D$ and control tasks. (B) Percentage of coherence averaged across the 179 neurons that were recorded in both the $\mathrm{CD}$ and the control tasks. (C) Distribution of the percentage of coherence for the 359 neurons with decision-related activity. Adapted with permission from Acuña and Pardo-Vazquez (2011).

Pardo-Vazquez, 2011). This result confirms that found by Romo et al. (2004) in the somatosensory discrimination task.

\section{PREMOTOR VENTRAL CORTEX AND THE EVALUATION OF THE DECISION PROCESS}

Depending on task demands, decisions may be postponed for later report and, in this case, they have to be maintained in working memory. Does PM cortex participate in maintaining these postponed decisions in working memory? Romo et al. have addressed this question in the PMm and PMv cortices during a postponed
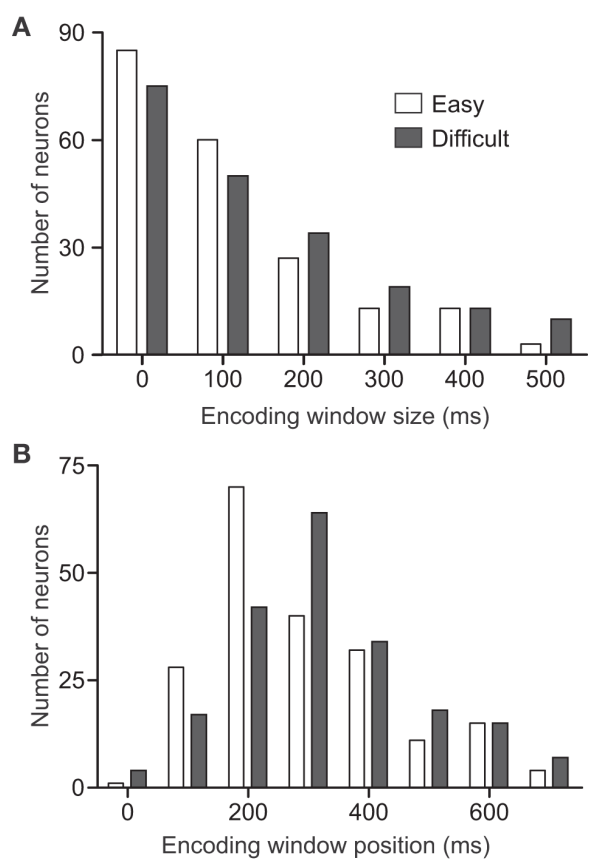

FIGURE 7 | The window size and position that maximize the coherence between neuronal and behavioral choices depend on the difficulty of the discrimination. Distributions of optimal window size (A) and optimal window position (B) as a function of the difficulty level for the 201 neurons for which the coherence between the behavioral and the neuronal choices is equal to or higher than $80 \%$. Adapted with permission from Acuña and Pardo-Vazquez (2011)

decision report period (Lemus et al., 2007, 2009). They have found that the activity of PMm neurons encodes both the result of the sensory evaluation (i.e., the choice) and past sensory information used to reach a decision during the somatosensory discrimination task (Lemus et al., 2007). Similar results were found in the PMv using the auditory discrimination task (Lemus et al., 2009; Figure 8). The main conclusions are that maintaining in WM the original information about the stimuli could serve to continuously update the postponed decision report and that during this period it is possible that the perceptual decision is evaluated and even corrected. This interpretation is consistent with the finding that behavioral performance is better when the decision report is postponed than when the decision is reported immediately after S2.

These neural representations have been studied along the cerebral cortex in a recent study (Hernandez et al., 2010), in which the activity of single cells from different cortical areas was recorded in monkeys performing a somatosensory discrimination task. It was found that perceptual decisions arise from the activity of neurons distributed across brain circuits and that these circuits represent the information necessary to evaluate the process.

\section{PERFORMANCE MONITORING IN INDIVIDUAL NEURONS DURING PERCEPTUAL DECISIONS}

The consequences of the behavioral decision affect all the components of the process and can modify future decisions (Gold and Shadlen, 2007); the decision process does not finish when 
the monkeys make a choice and execute the behavioral response. Therefore, the neural correlates of this stage of the decision process can be addressed by studying the neuronal activity after the behavioral responses in decision-related areas. As the PMv receives inputs from areas that encode information about the outcomes of previous decisions (Kurata, 1991; Hoover and Strick, 1993; Ghosh and Gattera, 1995; Takada et al., 2004; Boussaoud et al., 2005; Clower et al., 2005; Dancause et al., 2006), this issue was addressed in the choice-related PMv neurons, i.e., those that showed decision-related activity during the presentation of S2 (Pardo-Vazquez et al., 2008, 2009). In both the CD and the FDIR tasks neurons encoded: (a) the previous choices only, (b) the

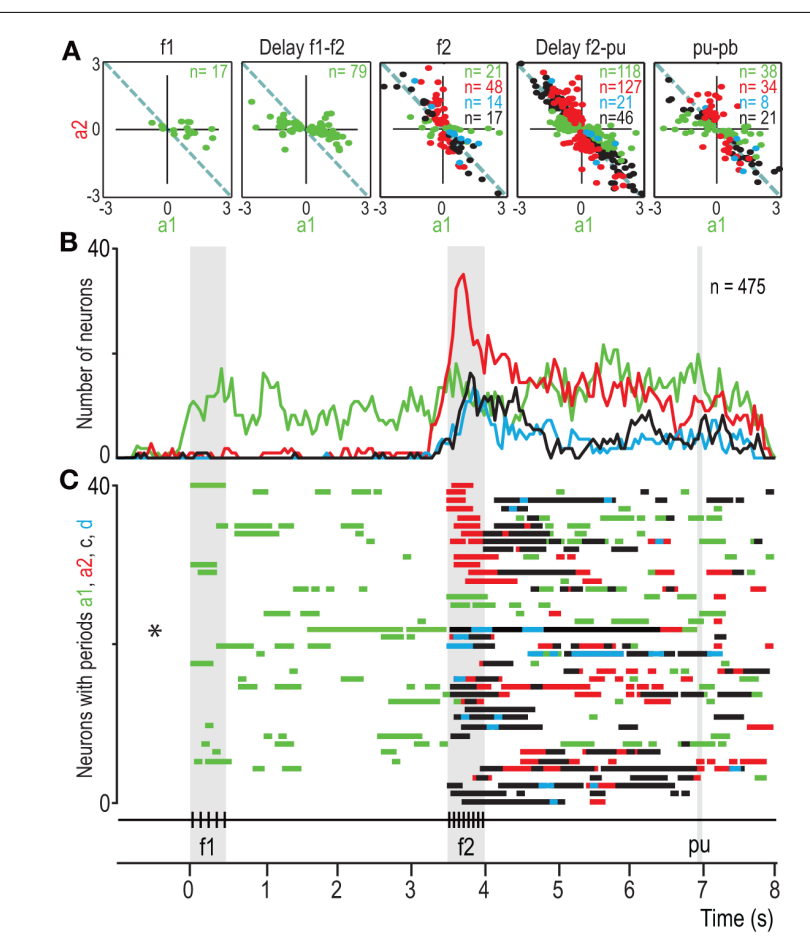

FIGURE 8 | Dynamics of PMv population responses during the acoustic flutter discrimination task. (A) Values of $a 1$ and $a 2$ coefficients for all neurons selected plotted B. For each point, at least one coefficient is significantly different from zero; $a 1$ and $a 2$ are the regression coefficients associated with the frequency of the first and second stimuli, respectively. Different plots are for various times in $A ; n=$ number of neurons. (B) Number of neurons with significant coefficients as a function of time. Green and red traces correspond to $a 1$ and $a 2$, respectively. Blue trace corresponds to neurons with both significant $a 1$ and $a 2$ coefficients of opposite signs, but significantly different magnitudes; these are partially differential (d) responses. Black trace corresponds to neurons with both significant $a 1$ and $a 2$ coefficients of opposite signs and statistically equal magnitude; these are fully differential (c) or categorical responses encoding $f 2-f 1$. (C) Bar graphs of 40 randomly selected neurons from the 475 neurons that contributed to (B). These bars indicate periods of responses encoding $f 1$ (green bars), $f 2$ (red bars), partially differential $f 2-f 1$ (blue bars), and fully differential or categorical responses encoding $f 2-f 1$ (black bars). Each line of bars represents the dynamics of the responses of one single neuron during the discrimination task. The dynamics of these coefficients was analyzed using a sliding window of 200 ms duration moving in steps of $100 \mathrm{~ms}$. pu, probe up; ku, key up; pb, push-button. Adapted with permission from Lemus et al. (2009). outcomes (correct or incorrect) only and (c) the previous choices and their outcomes (Figure 9). An important difference between the FDIR and the CD task is that, while in the former the correct orientation of S1 had to be retrieved by trial and error, in the later the stimulus is presented in each trial. Therefore, in the FDIR task the information about the retrieved $\mathrm{S} 1$ has to be combined with the information about the outcome of the decision while in the $\mathrm{CD}$ task this combination is not necessary and consequently, the representation of the orientation of S1 is not useful in this task. The PMv neurons reflect this difference in task requirements: after the behavioral response, and the feedback about the correctness of the previous choice, the PMv neurons encode the orientation of S1 that was compared with the orientation of S2 in the FDIR task but not in the CD task (Figure 9). Thus, the activity of the $\mathrm{PMv}$ neurons represents the information necessary to evaluate the previous decision process. We wish to emphasize that the fact that these neurons continued to mull over the past information used to reach a behavioral action suggests that the PMv neurons represent the information necessary to evaluate the previous decision process. This evaluation is necessary to learn from errors and adjust further behavior.

\section{PERFORMANCE MONITORING IN HUMANS: EVIDENCE FROM EVENT-RELATED POTENTIALS}

Given the importance of detecting errors, the neural bases of the system that encodes the outcomes of previous decisions have also been studied in humans using ERPs. In these studies two components of the ERPs related to error detection have been described. The first component, called error-related negativity (ERN; Gehring et al., 1993) or error negativity (Ne; Falkenstein et al., 1991), is defined as a negative deflection in the electric potential with a fronto-central scalp distribution that peaks about 80$100 \mathrm{~ms}$ after an erroneous response has been made. One property of the ERN is that it can be elicited following presentation of error feedback. This waveform, called the feedback error-related negativity (FRN), is a fronto-central negative deflection that occurs at approximately 250-350 ms after negative feedback stimuli (Miltner et al., 1997; Gehring and Willoughby, 2002; Holroyd and Coles, 2002). The second component, called error positivity (Pe; Falkenstein et al., 1991, 1995) is defined as a slow positive wave that usually follows the ERN, with centroparietal distribution, which peaks about 200-450 ms after an incorrect response. Source analyses and neuroimaging data have shown that these components are generated in the activity of neurons from the anterior cingulate cortex (ACC; Van Veen and Carter, 2002; Dehaene et al., 2004; Herrmann et al., 2004). Other localizations such as the basal ganglia and prefrontal cortex may also be involved in their generation (Gehring and Knight, 2000; Falkenstein et al., 2001).

During the last years the functional role of these ERPs has been studied using different behavioral tasks, including a visual discrimination task that is equivalent to the CD task.

The error-related potentials FRN and a positive deflection, that here we will refer to it as feedback related positivity (FRP), can be observed in Figure 10. (Pardo-Vazquez, Padron, FernandezRey and Acuña, Unpublished results). As both the ERPs and single cell data were obtained in equivalent tasks, it is possible to compare the temporal evolution of the outcomes-related 

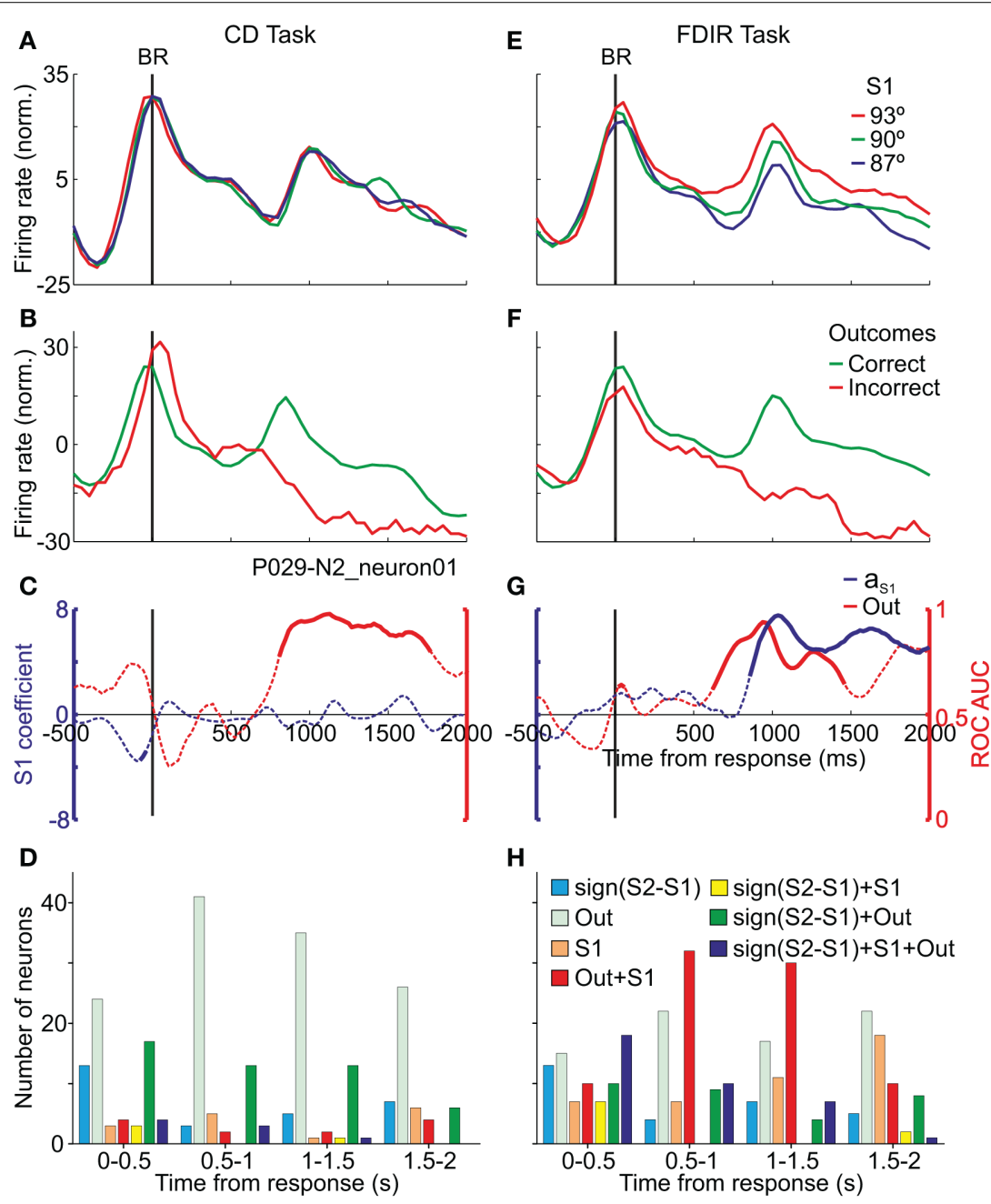

FIGURE 9 |After the monkey gave the behavioral response (BR) the same neuron encoded the outcomes (Out) and the memory traces of $S 1$ depending on the task. $(\mathbf{A}, \mathbf{E})$ Averaged firing rates sorted by $\mathrm{S} 1$; the memory traces are encoded in the FDIR task only. (B,F) Averaged firing rates sorted by correct and incorrect outcomes; in both tasks the same neuron differentiated correct from incorrect trials. $(\mathbf{C}, \mathbf{G})$ ROC AUC for correct vs.

activity. The difference between correct and incorrect trials is significant at $300-500 \mathrm{~ms}$ after the feedback is presented, both in the individual neurons recorded in the PMv cortex and in the humans' ERPs. Therefore, it is possible that the FRN and FRP are originated, at least in part, in the activity of those PMv neurons that encode the outcomes of previous decisions. Moreover, the results obtained in individual neurons of subhuman primates show that the brain encodes multiple types of information after the behavioral response and feedback presentation, including not only the outcomes of previous decisions but also the decisions themselves and the sensory information used to reach the decisions (Pardo-Vazquez et al., 2009). These complex neural representations have been revealed, in individual neurons, by the experimental manipulation of different behavioral variables of perceptual decision tasks. Therefore, this approach emerges as a promising method for revealing the contribution of the different cognitive incorrect trials and SLR $a_{\mathrm{S} 1}$ coefficient, as a function of time; continuous traces indicate significant values. (D,H) Number of neurons that carried significant information (ROC and SLR analysis) about the components of the decision in CD and FDIR task. Time intervals taken from the behavioral response. The same neurons can be represented in more than one time period. Adapted with permission from Pardo-Vazquez et al. (2009). processes that take place after the behavioral responses to the EEG activity.

\section{ROLE OF THE PMv CORTEX IN MAINTAINING INFORMATION IN WORKING MEMORY}

Taken together, the results obtained with perceptual discrimination tasks emphasize the role of the PM cortex in working memory and the representation of multiple features of the task during different time periods (Romo et al., 2004; Pardo-Vazquez et al., 2008, 2009; Lemus et al., 2009). The PMv neurons maintain in working memory information from different sources and for different purposes. These representations depend on task demands and also on the cognitive processes involved in each stage within the behavioral tasks (Pardo-Vazquez et al., 2009). The activity of PMv neurons represents, in different sensory modalities, the memory traces of sensory information, recently shown or recovered 


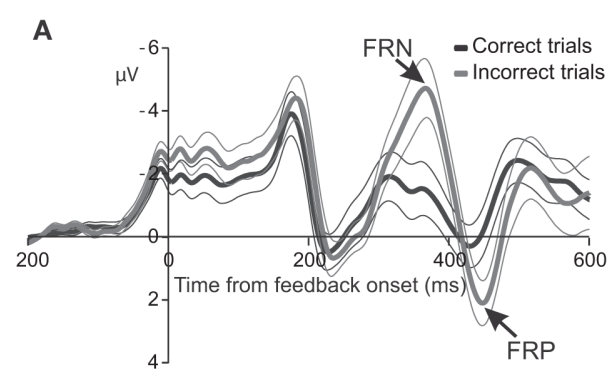

B
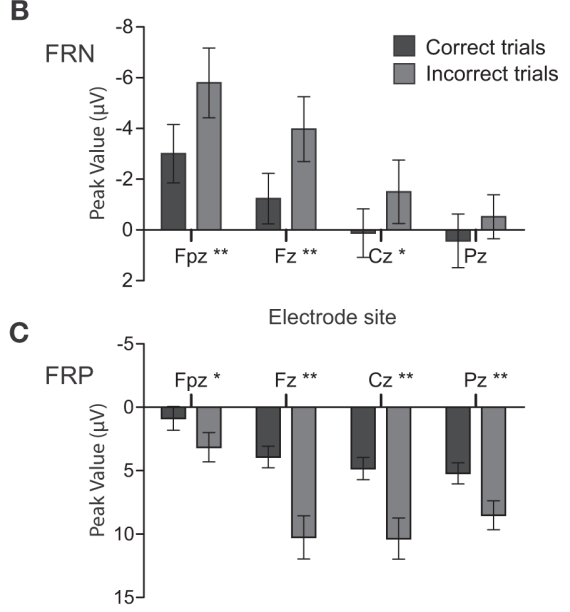

FIGURE 10 | Event-related potentials show significant differences between correct and incorrect decisions.

(A) Feedback-locked ERPs [mean $\pm(2 \times$ SEM $)$ ] at electrode Fpz following correct (black line) and incorrect (gray line) trials in a visual discrimination task $(n=13)$. Zero on abscissa indicates the feedback onset. Note that negative voltages are plotted upward by convention. (B,C) Mean peak amplitudes ( \pm SEM) of the FRN and FRP at electrodes $\mathrm{Fpz}, \mathrm{Fz}, \mathrm{Cz}$, and $\mathrm{Pz}$ for correct (black bars) and error (gray bars) trials. Significance was established using a two-tailed $t$-test at $p<0.01\left(^{*}\right)$ and $p<0.001\left(^{* *}\right)$.

from LTM, during the task period in which this information is used to reach a decision (Romo et al., 2004; Pardo-Vazquez et al., 2008, 2009; Lemus et al., 2009). During the postponed decision report, when the decision has to be maintained in WM until it is reported, the PMv neurons maintain a memory trace of the decision (Lemus et al., 2009). Similar results were obtained in the PMm neurons (Lemus et al., 2007). Behavioral data suggests that this task period may be used to evaluate the sensory information used to decide and, eventually, to correct the decision; it has been found that the activity of the PM neurons represents, during this period, the memory traces of the stimuli used to decide (Lemus et al., 2009). Finally, after the behavioral response, when the whole decision process is evaluated, the activity of the PMv neurons represents all the information needed for such evaluation, including the working memory traces of the previous decision and of the information used to decide (Pardo-Vazquez et al., 2008, 2009).

\section{FUNCTION OF THE PREMOTOR CORTEX REVISITED}

Early studies on the function of PM cortex were focused on motor aspects of behavior and used behavioral tasks designed to analyze the role of this area in the preparation and execution of movements (Wise, 1985; Passingham, 1993). Recent findings on the role of the PM cortex in decision-making have provided us with alternative interpretations about some of the motor functions attributed to this area, namely sensory guidance of movements (Petrides, 1982, 1985; Halsband and Passingham, 1985), maintenance of movements in working memory (Passingham, 1988), and associative learning (Sasaki and Gemba, 1982; Petrides, 1985, 1986; Halsband and Freund, 1990; Mitz et al., 1991).

The selection of movements based on sensory information has mainly been studied with conditional motor tasks and the main conclusion of these studies was that the PM cortex represents the selected movement when sensory information guides this selection (Wise, 1985; Passingham, 1993). Conditional motor tasks can also be interpreted as a form of perceptual decision as the subjects have to select the correct movement based on the available sensory information. Some of the results obtained with perceptual discrimination tasks indicate that the role of PM cortex goes far beyond representing the selected movement. Firstly, there are PMv neurons that represent the sensory evidence the monkeys use to reach a decision (Romo et al., 2004; Pardo-Vazquez et al., 2008, 2009; Lemus et al., 2009). Secondly, when the task implies the comparison of two stimuli presented sequentially, there are PMv neurons that represent not only the sign of the difference between the stimuli (i.e., the choice) but also its magnitude (Pardo-Vazquez et al., 2008; Acuña et al., 2010). Thirdly, when the same PMv neurons were evaluated in a motor task used as control (in which the motor component was the same as in the discrimination task but no discrimination was necessary) most of the neurons showed no significant differences as a function of the monkeys' movement (Pardo-Vazquez et al., 2008; Acuña et al., 2010).

The participation of the PM cortex in working memory has been studied with conditional motor tasks in which the subjects had to select one movement based on a sensory cue and to execute the movement immediately or after a delay period (Wise and Mauritz, 1985; Passingham, 1988). It has been found that neurons in the PM cortex show directional movement selectivity both in the conditions of the immediate and delayed tasks. From these results it has been concluded that the primary role of PM cortex would be to select the behavioral response and, once selected, hold it in memory until it was necessary (Passingham, 1988). The design of this kind of tasks makes it difficult to interpret the role of PM cortex in working memory; as in the behavioral tasks used each sensory cue is related to one movement, it could also be the case that PM neurons encode the sensory information during its presentation and maintain it in working memory during the delay or, as suggested by data from Lemus et al. (2009), these neurons could maintain both the decision and the sensory information. We favor this last interpretation because it is supported by the recent evidence that PM cortex participates in encoding both sensory and decision-related information and maintaining it in working memory (Romo et al., 2004; Pardo-Vazquez et al., 2008, 2009; Lemus et al., 2009).

Finally, early studies, both with lesions and single cell recordings, have also shown that the PM cortex participates in associative learning (Sasaki and Gemba, 1982; Petrides, 1985, 1986; Halsband and Freund, 1990; Mitz et al., 1991). It has been found that subjects 
with lesions in the PM area show learning deficits in association tasks. Commonly, subjects learn how to solve the task by trial and error and this implies that subjects have to relate the actions with their consequences. The results recently obtained while monkeys performed in discrimination tasks could help us to understand the role of PMv in learning (Pardo-Vazquez et al., 2008, 2009; Lemus et al., 2009; Acuña et al., 2010). There are neurons in PMv that encode previous decisions, their outcomes (Pardo-Vazquez et al., 2008; Acuña et al., 2010) and also the information used to reach a decision (Pardo-Vazquez et al., 2009; Acuña et al., 2010). The participation of the PMv in the evaluation of the decision process and of its consequences suggests that this area could be essential for shaping behavior on the basis of experience, through a learning process. Therefore, subjects with PM cortex lesions do not have the representations needed to evaluate the consequences of previous actions and the associative learning will be impaired.

An obvious question, in dealing with the PMv (F5/F4) area is the relationship between the neuronal activity obtained with discrimination tasks (Romo et al., 2004; Pardo-Vazquez et al., 2008,

\section{REFERENCES}

Acuña, C., and Pardo-Vazquez, J. L. (2011). Ventral premotor cortex neuronal activity matches perceptual decisions. Eur. J. Neurosci. 33, 2338-2348.

Acuña, C., Pardo-Vazquez, J. L., and Leboran, V. (2010). Decisionmaking, behavioral supervision and learning: an executive role for the ventral premotor cortex? Neurotox. Res. 18, 416-427.

Amiez, C., Kostopoulos, P., Champod, A. S., and Petrides, M. (2006). Local morphology predicts functional organization of the dorsal premotor region in the human brain. $J$. Neurosci. 26, 2724-2731.

Borra, E., Belmalih, A., Calzavara, R., Gerbella, M., Murata, A., Rozzi, S., and Luppino, G. (2008). Cortical connections of the macaque anterior intraparietal (AIP) area. Cereb. Cortex 18, 1094-1111.

Boudreau, M.-J., Brochier, T., Pare, M., and Smith, A. M. (2001). Activity in ventral and dorsal premotor cortex in response to predictable force-pulse perturbations in a precision grip task. J. Neurophysiol. 86, 1067-1078.

Boussaoud, D. (2001). Attention versus intention in the primate premotor cortex. Neuroimage 14, S40-S45.

Boussaoud, D., Tanne-Gariepy, J., Wannier, T., and Rouiller, E. M. (2005). Callosal connections of dorsal versus ventral premotor areas in the macaque monkey: a multiple retrograde tracing study. BMC Neurosci. 6, 67. doi: 10.1186/1471-2202-6-67

Boussaoud, D., and Wise, S. P. (1993). Primate frontal cortex: neuronal activity following attentional versus intentional cues. Exp. Brain Res. 95, 15-27.

Boyd, L. A., and Linsdell, M. A. (2009). Excitatory repetitive transcranial magnetic stimulation to left dorsal premotor cortex enhances motor consolidation of new skills. $B M C$ Neurosci. 10, 72. doi: 10.1186/14712202-10-72

Carey, J. R., Kimberley, T. J., Lewis, S. M., Auerbach, E. J., Dorsey, L., Rundquist, P., and Ugurbil, K. (2002). Analysis of fMRI and finger tracking training in subjects with chronic stroke. Brain 125, 773-788.

Cerri, G., Shimazu, H., Maier, M. A., and Lemon, R. N. (2003). Facilitation from ventral premotor cortex of primary motor cortex outputs to macaque hand muscles. $J$. Neurophysiol. 90, 832-842.

Clower, D. M., Dum, R. P., and Strick, P. L. (2005). Basal ganglia and cerebellar inputs to 'AIP'. Cereb. Cortex 15, 913-920.

Dancause, N., Barbay, S., Frost, S. B., Plautz, E. J., Stowe, A. M., Friel, K. M., and Nudo, R. J. (2006). Ipsilateral connections of the ventral premotor cortex in a new world primate. J. Comp. Neurol. 495, 374-390.

Dehaene, S., Molko, N., Cohen, L., and Wilson, A. J. (2004). Arithmetic and the brain. Curr. Opin. Neurobiol. 14, 218-224.

Deiber, M. P., Wise, S. P., Honda, M., Catalan, M. J., Grafman, J., and Hallett, M. (1997). Frontal and parietal networks for conditional motor-learning: a positron emission tomography study. J. Neurophysiol. 78, 977-991.

2009; Lemus et al., 2009) and the neuronal activity obtained during action observation (i.e., mirror activity) in F5 and F4 (reviewed in Rizzolatti and Sinigaglia, 2010). It is difficult to establish a direct relationship because of the different ways of obtaining the data and the uncertainty of the recording places. Albeit speculative, the encoding of a decision-making and evaluating process in the firing rate of the PMv neurons described here could also be embedded in the firing rate of the neurons active during action observation. Should this be the case, the encoding of the relevant sensory information (either current or from LTM) for deciding and acting and then evaluating that chosen action, might be at the service of action observation and communication.

\section{ACKNOWLEDGMENTS}

Work supported by grants from Ministerio de Ciencia e Innovación (MICINN) Spain to Carlos Acuña and Dirección Xeral de Investigación, Desenvolvemento e Innovación, Xunta de Galicia (INCITE) Spain to Carlos Acuña and Jose Fernandez-Rey. Work of Jose L. Pardo-Vazquez and Isabel Padron supported by INCITE post-doc and pre-doc grants, respectively.

Del Olmo, M. F., Cheeran, B., Koch, G., and Rothwell, J. C. (2007). Role of the cerebellum in externally paced rhythmic finger movements. J. Neurophysiol. 98, 145-152.

Denny-Brown, D., and Botterell, E. H. (1948). The motor functions of the agranular motor cortex. Res. Publ. Assoc. Res. Nerv. Ment. Dis. 27, 235-345.

Di-Pellegrino, G., and Wise, S. P. (1993). Visuospatial versus visuomotor activity in the premotor and prefrontal cortex of a primate. $J$. Neurosci. 13, 1227-1243.

Dum, R. P., and Strick, P. L. (1991). The origin of corticospinal projections from the premotor areas in the frontal lobe. J. Neurosci. 11,667-689.

Evarts, E. V. (1966). Pyramidal tract activity associated with a conditioned hand movement in the monkey. J. Neurophysiol. 29, 1011-1027.

Falkenstein, M., Hielscher, H., Dziobek, I., Schwarzenau, P., Hoormann, J., Sunderman, B., and Hohnsbein, J. (2001). Action monitoring, error detection, and the basal ganglia: an ERP study. Neuroreport 12, 157-161.

Falkenstein, M., Hohnsbein, J., and Hoormann, J. (1995). Event-related potential correlates of errors in reaction tasks. Electroencephalogr. Clin. Neurophysiol. Suppl. 44, 287-296.

Falkenstein, M., Hohnsbein, J., Hoormann, J., and Blanke, L. (1991). Effects of crossmodal divided attention on late ERP components. II. Error processing in choice reaction tasks. Electroencephalogr. Clin. Neurophysiol. 78, 447-455.
Freund, H.-J., and Hummelshein, H. (1985). Lesions of premotor cortex in man. Brain 108, 697-733.

Fridman, E. A., Hanakawa, T., Chung, M., Hummel, F., Leiguarda, R. C., and Cohen, L. G. (2004). Reorganization of the human ipsilesional premotor cortex after stroke. Brain 127, 747-758.

Fries, W., Danek, A., Scheidtmann, K., and Hamburger, C. (1993). Motor recovery following capsular stroke. Role of descending pathways from multiple motor areas. Brain $116(\mathrm{Pt}$ 2), 369-382.

Frost, S. B., Barbay, S., Friel, K. M., Plautz, E. J., and Nudo, R. J. (2003). Reorganization of remote cortical regions after ischemic brain injury: a potential substrate for stroke recovery. J. Neurophysiol. 89, 3205-3214.

Fulton, J. F. (1949). Physiology of the Nervous System. New York: Oxford University Press.

Fuster, J. (1997). The Prefrontal Cortex: Anatomy, Physiology, and Neuropsychology of the Prefrontal Lobe. Philadelphia: Lippincott-Raven.

Fuster, J. M., and Alexander, G. E. (1971). Neuron activity related to short-term memory. Science 173, 652-654.

Gehring, W. J., Goss, B., Coles, M. G. H., Meyer, D. E., and Donchin, E. (1993). A neural system for error detection and compensation. Psychol. Sci. 4, 385-390.

Gehring, W. J., and Knight, R. T. (2000). Prefrontal-cingulate interactions in action monitoring. Nat. Neurosci. 3 , 516-520. 
Gehring, W. J., and Willoughby, A. R. (2002). The medial frontal cortex and the rapid processing of monetary gains and losses. Science 295, 2279-2282.

Ghosh, S., and Gattera, R. (1995). A comparison of the ipsilateral cortical projections to the dorsal and ventral subdivisions of the macaque premotor cortex. Somatosens. Mot. Res. 12, 359-378.

Godschalk, M., Lemon, R. N., Kuypers, H. G., and Ronday, H. K. (1984). Cortical afferents and efferents of monkey postarcuate area: an anatomical and electrophysiological study. Exp. Brain Res. 56, 410-424.

Gold, J. I., and Shadlen, M. N. (2007). The neural basis of decision making. Annu. Rev. Neurosci. 30, 535-574.

Grafton, S. T., Fagg, A. H., and Arbib, M. A. (1998). Dorsal premotor cortex and conditional movement selection: a PET functional mapping study. J. Neurophysiol. 79, 1092-1097.

Graziano, M. S., and Aflalo, T. N. (2007). Mapping behavioral repertoire onto the cortex. Neuron 56, 239-251.

Graziano, M. S., Hu, X. T., and Gross, C. G. (1997). Visuospatial properties of ventral premotor cortex. J. Neurophysiol. 77, 2268-2292.

Green, D. M., and Swets, J. A. (1966). Signal Detection Theory and Psychophysics. New York: Wiley.

Halsband, U., and Freund, H. J. (1990). Premotor cortex and conditional motor learning in man. Brain $113(\mathrm{Pt}$ 1), 207-222.

Halsband, U., Ito, N., Tanji, J., and Freund, H. J. (1993). The role of premotor cortex and the supplementary motor area in the temporal control of movement in man. Brain $116(\mathrm{Pt}$ 1), 243-266

Halsband, U., and Passingham, R. (1982). The role of premotor and parietal cortex in the direction of action. Brain Res. 240, 368-372.

Halsband, U., and Passingham, R. E. (1985). Premotor cortex and the conditions for movement in monkeys (Macaca fascicularis). Behav. Brain Res. 18, 269-277.

He, S. Q., Dum, R. P., and Strick, P. L. (1993). Topographic organization of corticospinal projections from the frontal lobe: motor areas on the lateral surface of the hemisphere. $J$. Neurosci. 13, 952-980.

Hepp-Reymond, M., KirkpatrickTanner, M., Gabernet, L., Qi, H. X., and Weber, B. (1999). Contextdependent force coding in motor and premotor cortical areas. Exp. Brain Res. 128, 123-133.
Hepp-Reymond, M. C., Husler, E. J., Maier, M. A., and Ql, H. X. (1994). Force-related neuronal activity in two regions of the primate ventral premotor cortex. Can. J. Physiol. Pharmacol. 72, 571-579.

Hernandez, A., Nacher, V., Luna, R., Zainos, A., Lemus, L., Alvarez, M., Vazquez, Y., Camarillo, L., and Romo, R. (2010). Decoding a perceptual decision process across cortex. Neuron 66, 300-314.

Hernandez, A., Zainos, A., and Romo, R. (2002). Temporal evolution of a decision-making process in medial premotor cortex. Neuron 33 , 959-972.

Herrmann, M. J., Rommler, J., Ehlis, A. C., Heidrich, A., and Fallgatter, A. J. (2004). Source localization (LORETA) of the error-relatednegativity (ERN/Ne) and positivity (Pe). Brain Res. Cogn. Brain Res. 20 , 294-299.

Holroyd, C. B., and Coles, M. G. (2002). The neural basis of human error processing: reinforcement learning, dopamine, and the errorrelated negativity. Psychol. Rev. 109, 679-709.

Hoover, J. E., and Strick, P. L. (1993). Multiple output channels in the basal ganglia. Science 259, 819-821.

Hoshi, E., and Tanji, J. (2000). Integration of target and body-part information in the premotor cortex when planning action. Nature 408, 466-470.

Hoshi, E., and Tanji, J. (2002). Contrasting neuronal activity in the dorsal and ventral premotor areas during preparation to reach. J. Neurophysiol. $87,1123-1128$.

Iannilli, E., Gerber, J., Frasnelli, J., and Hummel, T. (2007). Intranasal trigeminal function in subjects with and without an intact sense of smell. Brain Res. 1139, 235-244.

Jacobsen, C. F. (1931). A study of cerebral function in learning. The frontal lobes. J. Comp. Neurol. 52, 271-340.

Jeannerod, M., Arbib, M. A., Rizzolatti, G., and Sakata, H. (1995). Grasping objects: the cortical mechanisms of visuomotor transformation. Trends Neurosci. 18, 314-320.

Kakei, S., Hoffman, D. S., and Strick, P. L. (2001). Direction of action is represented in the ventral premotor cortex. Nat. Neurosci. 4 1020-1025.

Keizer, K., and Kuypers, H. G. (1989). Distribution of corticospinal neurons with collaterals to the lower brain stem reticular formation in monkey (Macaca fascicularis). Exp. Brain Res. 74, 311-318.
Kelly, A. M. C., and Garavan, H. (2005). Human functional neuroimaging of brain changes associated with practice. Cereb. Cortex 15 , 1089-1102.

Kennard, M. A., Viets, H. R., and Fulton, J. F. (1934). The syndrome of the premotor cortex in man: impairment of skilled movements, forced grasping, spasticity, and vasomotor disturbance. J. Nerv. Ment. Dis. 80 93.

Kubota, K., and Niki, H. (1971). Prefrontal cortical unit activity and delayed alternation performance in monkeys. J. Neurophysiol. 34, 337-347.

Kurata, K. (1991). Corticocortical inputs to the dorsal and ventral aspects of the premotor cortex of macaque monkeys. Neurosci. Res. 12 , 263-280.

Kurata, K., and Hoffman, D. S. (1994). Differential effects of muscimol microinjection into dorsal and ventral aspects of the premotor cortex of monkeys. J. Neurophysiol. 71, 1151-1164.

Kurata, K., and Wise, S. P. (1988). Premotor and supplementary motor cortex in rhesus monkeys: neuronal activity during externally- and internally-instructed motor tasks. Exp. Brain Res. 72, 237-248.

Lang, W., Beisteiner, R., Lindinger, G., and Deecke, L. (1992). Changes of cortical activity when executing learned motor sequences. Exp. Brain Res. 89, 435-440.

Lemus, L., Hernandez, A., Luna, R., Zainos, A., Nacher, V., and Romo, R. (2007). Neural correlates of a postponed decision report. Proc. Natl. Acad. Sci. U.S.A. 104, 17174-17179.

Lemus, L., Hernandez, A., and Romo, R. (2009). Neural encoding of auditory discrimination in ventral premotor cortex. Proc. Natl. Acad. Sci. U.S.A. 106, 14640-14645.

Liu, Y., and Rouiller, E. M. (1999). Mechanisms of recovery of dexterity following unilateral lesion of the sensorimotor cortex in adult monkeys. Exp. Brain Res. 128, 149-159.

Lu, M. T., Preston, J. B., and Strick, P. L. (1994). Interconnections between the prefrontal cortex and the premotor areas in the frontal lobe. J. Comp. Neurol. 341, 375-392.

Luppino, G., Matelli, M., Camarda, R., and Rizzolatti, G. (1993). Corticocortical connections of area F3 (SMA-proper) and area F6 (pre-SMA) in the macaque monkey. J. Comp. Neurol. 338, 114-140.
Luppino, G., Murata, A., Govoni, P., and Matelli, M. (1999). Largely segregated parietofrontal connections linking rostral intraparietal cortex (areas AIP and VIP) and the ventral premotor cortex (areas F5 and F4). Exp. Brain Res. 128, 181-187.

Luppino, G., and Rizzolatti, G. (2000). The organization of the frontal motor cortex. News Physiol. Sci. 15, 219-224.

Luria, A. R. (1980). Higher Cortical Functions in Man. New York: Basic Books, Consultants Bureau.

Macmillan, N. A., and Creelman, C. D. (1991). Detection Theory. A User's Guide. Cambridge: Cambridge University Press.

Markowitsch, H. J., Irle, E., and Emmans, D. (1987). Cortical and subcortical afferent connections of the squirrel monkey's (lateral) premotor cortex: evidence for visual cortical afferents. Int. J. Neurosci. 37, 127-148.

Matelli, M., Camarda, R., Glickstein, M., and Rizzolatti, G. (1986). Afferent and efferent projections of the inferior area 6 in the macaque monkey. J. Comp. Neurol. 251, 281-298.

Matelli, M., Luppino, G., and Rizzolatti, G. (1985). Patterns of cytochrome oxidase activity in the frontal agranular cortex of the macaque monkey. Behav. Brain Res. 18, 125-136.

Mcfarland, N. R., and Haber, S. N. (2002). Thalamic relay nuclei of the basal ganglia form both reciprocal and nonreciprocal cortical connections, linking multiple frontal cortical areas. J. Neurosci. 22, 8117-8132.

Miltner, W., Braun, C., and Coles, M. (1997). Event-related brain potentials following incorrect feedback in a time-estimation task: evidence for a "generic" neural system for error detection. J. Cogn. Neurosci. 9, 788-798.

Mitz, A. R., Godschalk, M., and Wise, S. P. (1991). Learning-dependent neuronal activity in the premotor cortex: activity during the acquisition of conditional motor associations. $J$. Neurosci. 11, 1855-1872.

Mountcastle, V. B., Lynch, J. C., Georgopoulos, A., Sakata, H., and Acuña, C. (1975). Posterior parietal association cortex of the monkey: command functions for operations within extrapersonal space. $\mathrm{J}$. Neurophysiol. 38, 871-908.

Mushiake, H., Inase, M., and Tanji, J. (1991). Neuronal activity in the primate premotor, supplementary, and precentral motor cortex during visually guided and internally determined sequential movements. J. Neurophysiol. 66, 705-718. 
Newsome, W. T., Britten, K. H., and Movshon, J. A. (1989). Neuronal correlates of a perceptual decision. Nature 341, 52-54.

Pandya, D. D., and Barnes, C. L. (1987). "Architecture and connections of the frontal lobe," in The Frontal Lobes Revisited, ed. E. Perecman (New York: The IRBN Press), 41-72.

Pardo-Vazquez, J. L., Leboran, V., and Acuña, C. (2008). Neural correlates of decisions and their outcomes in the ventral premotor cortex. J. Neurosci. 28, 12396-12408.

Pardo-Vazquez, J. L., Leboran, V., and Acuña, C. (2009). A role for the ventral premotor cortex beyond performance monitoring. Proc. Natl. Acad. Sci. U.S.A. 106, 18815-18819.

Passingham, R. E. (1988). Premotor cortex and preparation for movement. Exp. Brain Res. 70, 590-596.

Passingham, R. E.(1993). The Frontal Lobes and Voluntary Action. Oxford: Oxford University Press.

Passingham, R. E., and Toni, I. (2001). Contrasting the dorsal and ventral visual systems: guidance of movement versus decision making. $\mathrm{Neu}$ roimage 14, S125-S131.

Petrides, M. (1982). Motor conditional associative-learning after selective prefrontal lesions in the monkey. Behav. Brain Res. 5, 407-413.

Petrides, M. (1985). Deficits on conditional associative-learning tasks after frontal- and temporal-lobe lesions in man. Neuropsychologia 23, 601-614.

Petrides, M. (1986). The effect of periarcuate lesions in the monkey on the performance of symmetrically and asymmetrically reinforced visual and auditory go, no-go tasks. J. Neurosci. 6, 2054-2063.

Petrides, M., Cadoret, G., and Mackey, S. (2005). Orofacial somatomotor responses in the macaque monkey homologue of Broca's area. Nature 435, 1235-1238.

Petrides, M., and Milner, B. (1982). Deficits on subject-ordered tasks after frontal- and temporal-lobe lesions in man. Neuropsychologia 20, 249-262.

Preuss, T. M., Stepniewska, I., and Kaas, J. H. (1996). Movement representation in the dorsal and ventral premotor areas of owl monkeys: a microstimulation study. J. Comp. Neurol. 371, 649-676.

Rizzolatti, G., and Craighero, L. (2004). The mirror-neuron system. Annu. Rev. Neurosci. 27, 169-192.

Rizzolatti, G., and Fadiga, L. (1998). Grasping objects and grasping action meanings: the dual role of monkey rostroventral premotor cortex (area F5). Novartis Found. Symp. 218, 81-95.

Rizzolatti, G., and Luppino, G. (2001). The cortical motor system. Neuron 31, 889-901.

Rizzolatti, G., Luppino, G., and Matelli, M. (1998). The organization of the cortical motor system: new concepts. Electroencephalogr. Clin. Neurophysiol. 106, 283-296.

Rizzolatti, G., and Matelli, M. (2003). Two different streams form the dorsal visual system: anatomy and functions. Exp. Brain Res. 153, 146-157.

Rizzolatti, G., and Sinigaglia, C. (2010). The functional role of the parietofrontal mirror circuit: interpretations and misinterpretations. Nat. Rev. Neurosci. 11, 264-274.

Roca-Pardiñas, J., Cadarso-Suárez, C., Pardo-Vazquez, J. L., Leboran, V., Molenberghs, G., Faes, C., and Acuña C. (2011). Assessing neural activity related to decision-making through flexible odds ratio curves and their derivatives. Stat. Med. 30, 1695-1711.

Roland, P. E., Larsen, B., Lassen, N. A., and Skinhoj, E. (1980). Supplementary motor area and other cortical areas in organization of voluntary movements in man. J. Neurophysiol. 43, 118-136.

Romo, R., Hernandez, A., and Zainos, A. (2004). Neuronal correlates of a perceptual decision in ventral premotor cortex. Neuron 41, 165-173.

Romo, R., Merchant, H., Zainos, A., and Hernandez, A. (1997). Categorical perception of somesthetic stimuli: psychophysical measurements correlated with neuronal events in primate medial premotor cortex. Cereb. Cortex 7, 317-326.

Romo, R., Ruiz, S., Crespo, P., Zainos, A., and Merchant, H. (1993). Representation of tactile signals in primate supplementary motor area. J. Neurophysiol. 70, 2690-2694.

Romo, R., and Schultz, W. (1987). Neuronal activity preceding selfinitiated or externally timed arm movements in area 6 of monkey cortex. Exp. Brain Res. 67, 656-662.

Romo, R., and Schultz, W. (1992). Role of primate basal ganglia and frontal cortex in the internal generation of movements. III. Neuronal activity in the supplementary motor area. Exp. Brain Res. 91, 396-407.

Rouiller, E. M., Tanne, J., Moret, V., and Boussaoud, D. (1999). Origin of thalamic inputs to the primary, premotor, and supplementary motor cortical areas and to area 46 in macaque monkeys: a multiple retrograde tracing study. J. Comp. Neurol. 409, 131-152.

Sasaki, K., and Gemba, H. (1982). Development and change of cortical field potentials during learning processes of visually initiated hand movements in the monkey. Exp. Brain Res. 48, 429-437.

Schell, G. R., and Strick, P. L. (1984). The origin of thalamic inputs to the arcuate premotor and supplementary motor areas. J. Neurosci. 4, 539-560.

Schluter, N. D., Rushworth, M. F., Passingham, R. E., and Mills, K. R. (1998). Temporary interference in human lateral premotor cortex suggests dominance for the selection of movements. A study using transcranial magnetic stimulation. Brain 121(Pt 5), 785-799.

Schubotz, R. I., and Von Cramon, D. Y. (2001). Functional organization of the lateral premotor cortex: fMRI reveals different regions activated by anticipation of object properties, location and speed. Brain Res. Cogn. Brain Res. 11, 97-112.

Schubotz, R. I., Von Cramon, D. Y., and Lohmann, G. (2003). Auditory what, where, and when: a sensory somatotopy in lateral premotor cortex. Neuroimage 20, 173-185.

Seitz, R. J., Hoflich, P., Binkofski, F., Tellmann, L., Herzog, H., and Freund, H. J. (1998). Role of the premotor cortex in recovery from middle cerebral artery infarction. Arch. Neurol. 55, 1081-1088.

Shadmehr, R., and Holcomb, H. H. (1997). Neural correlates of motor memory consolidation. Science 277 , 821-825.

Shimazu, H., Maier, M. A., Cerri, G. Kirkwood, P. A., and Lemon, R. N. (2004). Macaque ventral premotor cortex exerts powerful facilitation of motor cortex outputs to upper limb motoneurons. J. Neurosci. 24, 1200-1211.

Takada, M., Nambu, A., Hatanaka, N., Tachibana, Y., Miyachi, S., Taira, M., and Inase, M. (2004). Organization of prefrontal outflow toward frontal motor-related areas in macaque monkeys. Eur. J. Neurosci. 19, 3328-3342.

Travis, A. M. (1955). Neurological deficiencies after ablation of the precentral motor area in Macaca mulatta. Brain 78, 155-173.

Van Veen, V., and Carter, C. S. (2002). The anterior cingulate as a conflict monitor: fMRI and ERP studies. Physiol. Behav. 77, 477-482.
Vazquez, P., Cano, M., and Acuña, C. (2000). Discrimination of line orientation in humans and monkeys. $J$. Neurophysiol. 83, 2639-2648.

Walshe, F. M. R. (1935). On the "syndrome of the premotor cortex" (Fulton) and the definition of the terms "Premotor" and "Motor": with a consideration of Jackson's views on the cortical representation of movements. Brain 58, 49-80.

Weinrich, M., and Wise, S. P. (1982). The premotor cortex of the monkey. $J$. Neurosci. 2, 1329-1345.

Weinrich, M., Wise, S. P., and Mauritz, K. H. (1984). A neurophysiological study of the premotor cortex in the rhesus monkey. Brain 107, 385-414.

Wise, S. P. (1985). The primate premotor cortex: past, present, and preparatory. Annu. Rev. Neurosci. 8, 1-19.

Wise, S. P., Boussaoud, D., Johnson, P. B., and Caminiti, R. (1997). Premotor and parietal cortex: corticocortical connectivity and combinatorial computations. Annu. Rev. Neurosci. 20, 25-42.

Wise, S. P., and Mauritz, K. H. (1985). Set-related neuronal activity in the premotor cortex of rhesus monkeys: effects of changes in motor set. Proc. R. Soc. Lond. B Biol. Sci. 223, 331-354.

Xiao, J., Padoa-Schioppa, C., and Bizzi, E. (2006). Neuronal correlates of movement dynamics in the dorsal and ventral premotor area in the monkey. Exp. Brain Res. 168, 106-119.

Conflict of Interest Statement: The authors declare that the research was conducted in the absence of any commercial or financial relationships that could be construed as a potential conflict of interest.

Received: 01 June 2011; paper pending published: 18 July 2011; accepted: 26 August 2011; published online: 27 September 2011.

Citation: Pardo-Vazquez JL, Padron I, Fernandez-Rey $J$ and Acuña C (2011) Decision-making in the ventral premotor cortex harbinger of action. Front. Integr. Neurosci. 5:54. doi: 10.3389/fnint.2011.00054

Copyright (C) 2011 Pardo-Vazquez, Padron, Fernandez-Rey and Acuña. This is an open-access article subject to a non-exclusive license between the authors and Frontiers Media SA, which permits use, distribution and reproduction in other forums, provided the original authors and source are credited and other Frontiers conditions are complied with. 